\title{
A comparative Study of Tonification and Sedation in Moxibustion using Computer Method
}

\section{Miao Liu ( $\nabla$ liumiao@sues.edu.cn )}

Shanghai University of Engineering Science https://orcid.org/0000-0001-9762-9523

\section{S.K. Kauh}

Seoul National University

\section{Sabina Lim}

Kyung Hee University

\section{Research}

Keywords: Moxibustion, Finite Element Method, Skin Tissue, Bioheat Transfer, Thermal Damage, Tonification, Sedation

Posted Date: January 21st, 2022

DOI: https://doi.org/10.21203/rs.3.rs-903359/v2

License: (9) This work is licensed under a Creative Commons Attribution 4.0 International License. Read Full License 


\section{A comparative Study of Tonification and Sedation in Moxibustion using Computer Method}

\author{
Miao Liu \\ School of Mechanical and Aerospace Engineering \\ Seoul National University \\ Seoul, S.Korea \\ Liumiao1@snu.ac.kr
}

\author{
Sang Ken Kauh \\ School of Mechanical and Aerospace Engineering \\ Seoul National University \\ Seoul, S.Korea
}

\begin{abstract}
Moxibustion is an important and widely used treatment method in traditional medical science. Two methods of moxibustion exist: tonification and sedation. Although these two methods have been used for thousands of years, there still has been no scientific comparative study of their effects on the skin until now. Thus, this paper aims to use a mathematical method to study moxibustion methods in skin tissue to demonstrate a difference of thermomechanics between tonification and sedation. In this paper, the temperature, burn damage, and thermal stress distributions in the skin tissue is analyzed and the results showed that the thermomechanical behavior of skin tissue between tonification and sedation is in accordance to ancient literatures of traditional medicine. Therefore, the comparative study of heat transfer and related thermomechanics in skin tissue during these treatments thermomechanics to tonification and sedation is great importance and helpful in exploring moxibustion instruments and their standardization contribute to the further developments of these medical applications.
\end{abstract}

Keywords-Moxibustion; Finite Element Method; Skin Tissue; Bioheat Transfer; Thermal Damage; Tonification, Sedation

\section{INTRODUCTION}

Although moxibustion has been used throughout Asia for thousands of years, and plays an important role in the traditional medical systems of China, Japan, Korea, Vietnam, Tibet, and Mongolia, there still has been no scientific comparative study of their effects on the skin until now. Therefore, in this paper, a mathematical method with finite element analysis is adopted to compare the thermomechanics of skin tissue to tonification and sedation treatment, where the temperature, burn damage, and thermal stress distributions in the skin tissue are analyzed. Moreover, a standardized method to measure the temperature of tonification and sedation were done to get the burning temperature.[1]

Moxibustion is a traditional medicine therapy using moxa or mugwort herb ground up to fluff that burned on an acupuncture point, and there are two approaches of moxibustion treatment proposed by Chinese ancestors: tonification and sedation. These approaches are described in the classical book Miraculous Pivot of The Yellow Emperor's Internal Classic_-Ling Shu (Miraculous Pivot, also known as
Canon of Acupuncture). Tonification is used for reinforcement and involves allowing a slow burning cone to burn out by itself; although the heat is weak, it is persistent and substantial. If this supplementing technique is used, the moxa cone is left alone after it is ignited. It must be allowed to burn gradually and eventually burn out. Then the point is pressed with the hand to concentrate the qi and make the warming persistent. Sedation is used for reduction. The process involves blowing on the moxa cone. Although the heat is intense, it is temporary and short. If this drainage technique is used, the ignited moxa must be blown upon to increase its speed of burning; then, it either burns out or is removed when it becomes too hot. Unlike tonification, the point is not pressed. Instead, the pathogenic qi is scattered externally.[2]

In this paper, we first introduce the mathematical method. Secondly, we provide a standardized method to measure the temperature of tonification and sedation. Finally, based on the temperature of the burning moxa cone, the results of analysis using a finite element method are presented.

\section{METHOD OF APPROACH}

Moxibustion requires placing a moxa cone directly on the skin. As the moxa cone burns, the temperature rises on the skin surface; thus, the heat generated on the skin produces physical and chemical stimuli that cause the body to experience pain. Therefore, the study of bioheat transfer, thermal damage and thermal stress in the skin tissue is helpful to understand the mechanism of tonification and sedation. However, the current temperature measurement technology can only measure at fixed points; thus, in this paper a mathematical model of skin tissue experiencing tonification and sedation is used and described below.[4]

\section{A. Skin structure}

The skin is the largest multi-layer organ in the body. The structure of skin is extremely complicated, but it is generally made up of four layers: the stratum corneum, the epidermis, the dermis, and the hypodermis, as shown in Fig.1.

According to the skin structure, in this paper, an ideal skin layer is used, as shown in Fig.2. Moxibustion mathematical model were studied based on these four layers, Each layer has a different thickness: the thickness of the stratum corneum is $0.02 \mathrm{~mm}$, the thickness of the epidermis is $0.08 \mathrm{~mm}$, the thickness of the dermis is $1.5 \mathrm{~mm}$, and the thickness of the 
subcutaneous fat is $4.4 \mathrm{~mm}$. While blood perfusion only occurs in the dermis layer, metabolic heat generation occurs in all four layers.

\section{B. Bioheat transfer}

We used the classical, extensively applied Pennes' bioheat equation ${ }^{12}$ to describe the heat transfer of direct moxibustion:

$$
\rho \frac{\partial \mathrm{T}}{\partial \mathrm{t}}=\mathrm{k} \nabla^{2} \mathrm{~T}+\overline{\omega_{b}} \rho_{b} c_{b}\left(\mathrm{~T}_{\mathrm{a}}-\mathrm{T}\right)+q_{\text {met }}+\mathrm{q}_{\mathrm{ext}}
$$

where $\rho$ is the density of skin tissue; $k$ is the thermal conductivity of skin tissue; $\overline{\omega_{b}}$ is the blood perfusion rate; $\rho_{b}$ is the density of blood; $\mathrm{c}_{\mathrm{b}}$ is the specific heat of blood; $\mathrm{T}_{\alpha}$ is the temperature of blood; $\mathrm{T}$ is the temperature of skin tissue; $\mathrm{q}_{\text {met }}$ is the metabolic heat generation in the skin tissue; and $\mathrm{q}_{\text {ext }}$ is the heat generation due to external heating sources.[5]

\section{Thermal damage}

Currently, the Arrhenius burn integration proposed by Henriques and Moritz (1947) $)^{13,14}$ is commonly used to describe thermal damage as the following:

$$
\begin{aligned}
& \mathrm{k}(\mathrm{T})=\frac{\partial \Omega}{\partial \mathrm{t}}=\mathrm{A} \exp \left(-\frac{\mathrm{E}_{\mathrm{a}}}{\mathrm{RT}}\right) \\
& \text { or equivalently: } \\
& \Omega=\int_{0}^{\mathrm{t}} \mathrm{A} \exp \left(-\frac{\mathrm{E}_{\mathrm{a}}}{\mathrm{RT}}\right)
\end{aligned}
$$

Where $\mathrm{k}$ is the rate of protein denaturation; $\mathrm{t}$ is the exposure time; $\mathrm{T}$ is the absolute temperature $(\mathrm{K}) ; \mathrm{A}$ is a material parameter equivalent to a frequency factor $\left(\mathrm{s}^{-1}\right)$; $\mathrm{E}_{\mathrm{\alpha} \alpha}$ is the activation energy $(\mathrm{J} / \mathrm{mole})$; and $\mathrm{R}$ is the universal gas constant $\left(8.314 \mathrm{~J}-\mathrm{mol}^{-1} \mathrm{~K}^{-1}\right)$.

In accordance to previous studies, an activation energy equals $E_{\alpha \underline{\alpha}}$ to $E_{\alpha}=6.28 \times 10^{5} \mathrm{~J} / \mathrm{mol}$ and a frequency factor $A$ equals to $\mathrm{A}=3.1 \times 10^{98} \mathrm{~s}^{-1}$ were used in this paper for thermal damage of skin tissue.

\section{Thermal stress}

Skin can be considered as a mechanical structure. During moxibustion treatment, the skin experiences mechanical stimulation caused by thermal stress. Here the skin is treated as a laminated composite structure, in which each layer is assumed to be uniform with linear thermo-elastic properties. We calculated the thermal stress value using the finite element method software COMSOL based on coupling the data obtained from the bioheat transfer.

\section{TEMPERATURE MEASUREMENT}

To study the thermomechanics of tonification and sedation, the temperature of burning moxa should be measured. The burning scope and temperature curve of moxa can be acquired from ideal and standardized burning experiments on tonification and sedation, respectively. To measure the burning temperature of a moxa cone, we used experimental equipment with a hot plate managed by a control system inside the chamber.[3]

\section{A. Materials}

To test this equipment, we used "KangHwa" moxa made by Ehwadang in the year 2007, and to make the moxa cone for direct moxibustion, we used barley that was produced in Korea. Their scientific name is Hordeum vulgare var. hexstichon

The moxa cone was burned on the hot plate in the chamber of this system. The fan and plate temperature were controlled with a microcontroller. A thermocouple was used to link the control system. Finally, the data were transmitted to the computer. The system is shown in Fig.3.

\section{B. Experimental Approach}

We used a vernier caliper made by Mitutoyo Co., Japan, with an accuracy of $0.01 \mathrm{~mm}$ to measure the size of ten prepared barley moxa cones and ten prepared jujube moxa cones. We also used a Sartorius CP224S scale made by Kinematica AG, Germany, to weigh the 20 prepared samples.

To measure the burning temperature during tonification, a thermocouple was fixed to the hot plate with a constant temperature of $34^{\circ} \mathrm{C}$ using aluminum tape. Then a conglutinated barley moxa cone was linked to the thermocouple by saliva. The moxa cone's apex was lighted with a joss stick, and then the temperature was measured. A total of 20 samples were prepared. The temperature was measured every second after the moxa cone was lighted. It was measured for 40 seconds for the barley moxa cone.

For sedation, air was blown on the moxa cone from $20 \mathrm{~cm}$ away every two seconds. The experiment lasted only 30 seconds. The data statistics are shown in Table 1.

According to the results from our previous experiment, the burning temperature generated by sedation was approximately twice that of tonification, but sedation required much more time to reach the peak temperature.

\section{RESUlTS}

It is known that many types of diversified sensor cells are distributed in the dermis layer. Thus, our experiment focused on this layer to analyze the thermomechanics of the epidermisdermis interface (ED interface) to compare tow method of moxibustion. For the thermomechanics analysis, two groups of data from the previous burning temperature experiments were used and Tables 2 and 3 display the parameter values of each skin layer.

\section{A. Tonification analysis}

We placed the heat source onto a piece of skin with an area equal to that of the bottom of the barley moxa cone, heated the skin for 20 seconds and left the skin for an additional 5 seconds to cool down. Then we observed changes in the skin temperature and calculated the thermal damage and thermal stress. Fig.4 (a) and (b) show the temperature distribution in the skin during heating and cooling $(\mathrm{t}=5 \quad \mathrm{~s})$ and the temperature distribution at the ED interface over time, respectively. The corresponding thermal damage and thermal stress fields can be calculated using the obtained temperature fields (see Fig.5 and 6)

\section{B. Sedation analysis}

We placed the heat source onto a piece of skin with an area equal to that of the bottom of the barley moxa cone, heated the skin for 15 seconds, and then removed the heat source. Finally, air with a temperature of $20^{\circ} \mathrm{C}$ was used for 5 seconds to cool 
the skin. Temperature changes in the skin were recorded, and the thermal damage and thermal stress were calculated. Fig.7 (a) and (b) show the temperature distribution in the skin after heating and cooling $(\mathrm{t}=5 \mathrm{~s})$ and the temperature distribution at the ED interface over time, respectively.The corresponding thermal damage and thermal stress fields can be calculated using the obtained temperature fields (Fig. 8 and 9).

\section{Summary}

For thermal transfer, as the fig.4(a) and 7(a) shows, sedation penetrated the skin much deeper than tonification. Also, as the fig.4(b) and 7(b) shows, the peak temperature in ED interface of sedation is higher than that of tonification. In the procedure of cooling, heat will be continuously transferred into skin tissue by tonification method. But, using sedation, heat will be diffused into air. For thermal damage, as the fig.5 and 8 shows, the damage caused by sedation is larger than that by tonification. For thermal stress, as the fig.6(a) and 9(a) shows, distributions in the skin tissue caused by sedation are similar with that by tonification. Besides, thermal stress is also similar between sedation and tonification before cooling. However, as the fig.6(b) and 9(b) shows, thermal stress in ED interface caused by sedation becomes much larger than that by tonification after cooling.

Thus, in view of analysis above, sedation has much larger stimulus to skin tissue than tonification, which is in accordance to ancient literatures of traditional medicine.

\section{CONCLUSION}

The purpose of this paper is to use mathematical models to compare the thermomechanics produced by tonification and sedation. Although these two methods have been used for thousands of years, there has been no scientific comparative study of their effects on the skin until now, so that it limited the development of tonification and sedation. Therefore, the study of this paper about heat transfer and related thermomechanics in skin tissue is of great importance and can contribute to the application of moxibustion, improvements in moxibustion treatment, and standardization of moxibustion.

It is known that moxibustion works by stimulating the acupuncture point of the skin tissue, and the distinction between Tonification and Sedation is just the stimulus. After obtaining the temperature properties of tonification and sedation, the stimulus of tonification and sedation is analyzed by studying the combustion temperature for skin irritation and skin stimulations using a mathematical model. The result shows that the stimulus of tonification and sedation is in accordance to ancient literatures of traditional medicine. Therefore, with a mathematical model, the stimulus of tonification and sedation can be understood and used to further develop the tool of moxibustion.

This paper has several limitations. Its main deficiency is the assumption that the skin tissue properties are constant. Collagen is the major component of skin, and its thermal and mechanical properties vary with temperature. However, there have been relatively few studies regarding these changes, which significantly limits research on moxibustion treatment. Moreover, Ikuko Nishitani has suggested that other biochemical responses such as chemical stimuli are generated during moxibustion treatment.[13]
Table 1 The combustion temperature of direct moxibustion by tonification and sedation(Each value is mean $\pm \mathrm{SD}$ )

\begin{tabular}{ccc}
\hline \hline & Tonification & Sedation \\
\hline Peak temperature $\left({ }^{\circ} \mathrm{C}\right)$ & $129.1 \pm 33.3$ & $240.2 \pm 46.4$ \\
Peak time $(\mathrm{sec})$ & $11.6 \pm 2.6$ & $15.3 \pm 3.2$
\end{tabular}

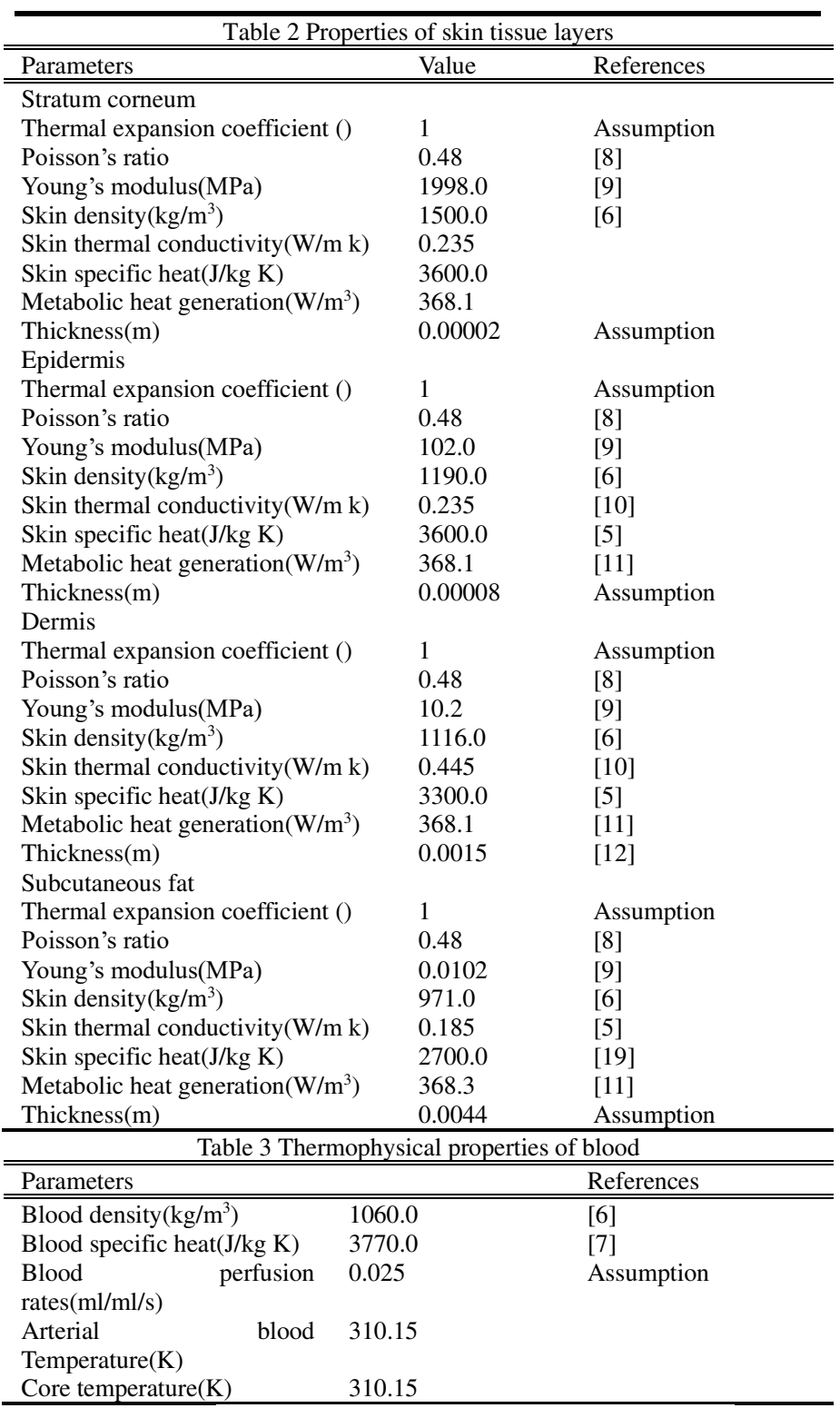

Core temperature $(\mathrm{K})$

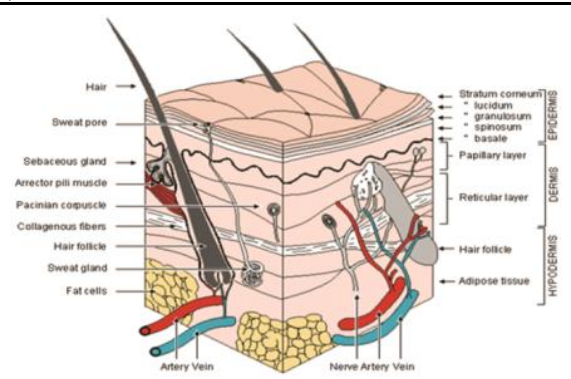

Figure.1 Section of smooth skin (Redrawn from Ebling, Eady, and Leigh, 1992 
Heat of Direct

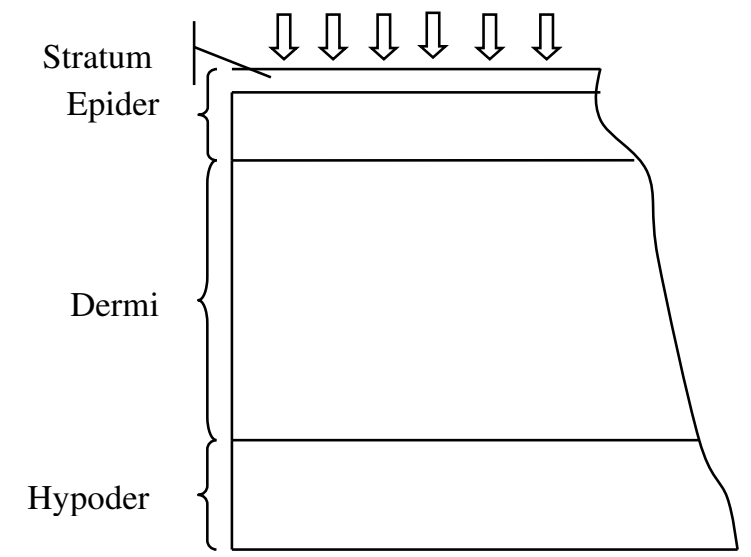

Figure.2 Ideal skin layers for thermomechanics study

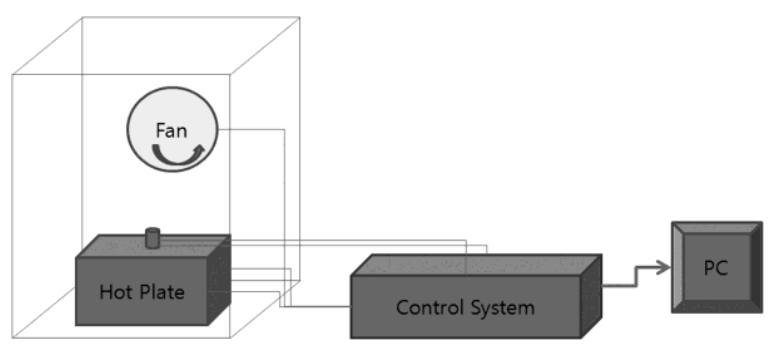

Figure.3 Temperature measurement system

a

b

Figure.4 (a) Temperature distribution in skin at (t=10 s, $13 \mathrm{~s}, 16 \mathrm{~s}$, and $20 \mathrm{~s})$; (b) temperature history at the ED interface.

a

\section{b}

Figure.5 (a) Thermal damage distribution in skin at (t=10 s, $13 \mathrm{~s}, 16 \mathrm{~s}$, and 20

a

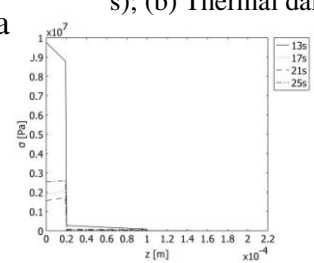

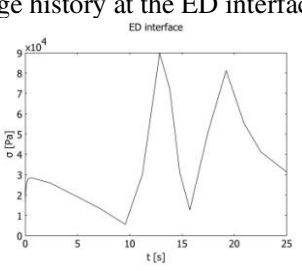

Figure.6 (a) Thermal stress distribution in skin at ( $\mathrm{t}=10 \mathrm{~s}, 13 \mathrm{~s}, 16 \mathrm{~s}$, and 20 s); (b) Thermal stress history at the ED interface.

a

b

Figure.7 (a) Temperature distribution in skin at $(\mathrm{t}=8 \mathrm{~s}, 12 \mathrm{~s}, 16 \mathrm{~s}$, and $22 \mathrm{~s})$; (b) temperature history at the ED interface.

a

b

Figure.8 (a) Thermal damage distribution in skin at ( $\mathrm{t}=8 \mathrm{~s}, 12 \mathrm{~s}$, and 22s); (b) Thermal damage history at the ED interface.

a

b

Figure.9 (a) Thermal stress distribution in skin at ( $\mathrm{t}=8 \mathrm{~s}, 12 \mathrm{~s}, 16 \mathrm{~s}$, and 22s); (b) Thermal stress history at the ED interface.

\section{REFERENCES}

[1]. David P.Q. Zhu. The role of Moxibustion in Traditional Chinese Acupuncture. American Journal of Acupuncture. 1984;12(2):125-132.

[2]. Suobin Kang. Classical Acupuncture \& Moxibusion. Seoul. Iljungsa Press. 2000.

[3]. Geo-Mok Lee, Geon-Mok Lee, Yoo-Jin Hwang. Experimental Study on the Thermodynamic Characteristics of Commercial Small-size Moxa Combustion. The Journal of Korean Acupuncture \& Moxibustion Society Vol.18 No.6 (2002)

[4]. F.Xu, T.J.Lu, K.A.Seffen. Biothermomechanical behavior of skin tissue. Acta Mech Sin 2008 24:1-23

[5]. Henriques, F.C., Moritz, A.R.: Studies of thermal injury, 1. The conduction of heat to and through skin and the temperatures attained therein. A theoretical and an experimental investigation. Am. J. Pathol. 23, 531-549 (1947). 19

[6]. Duck, F.A.: Physical Properties of Tissue:AComprehensive Reference Book. Academic Press, London (1990)

[7]. Torvi, D.A., Dale, J.D.: A finite element model of skin subjected to a flash fire. J. Biomech. Eng. 116(3), 250-255 (1994)

[8]. Delalleau, A., Josse, G., Lagarde, J.M., et al.: Characterization of the mechanical properties of skin by inverse analysis combined with the indentation test. J. Biomech. 39(9), 1603-1610 (2006)

[9]. Hendriks, F.M., Brokken, D., Oomens, C.W., et al.: The relative contributions of different skin layers to the mechanical behavior of human skin in vivo using suction experiments. Med. Eng. Phys. 28(3), 259-266 (2006)

[10]. Elkins, W., Thomson, J.G.: Instrumented Thermal Manikin. Acurex Corporation, Aerotherm Division Report AD-781, p. 176 (1973)

[11]. Roetzel,W., Xuan,Y.: Transient response of the human limb to an external stimulus. Int. J.Heat Mass Transf. 41(1), 229-239 (1998)

[12]. Dahan,S., Lagarde,J.M., Turlier,V., et al: Treatment of neck lines and forehead rhytids with a nonablative 1540-nm Er: glass laser: a controlled clinical study combined with the measurement of the thickness and the mechanical properties of the skin. Dermatol. Surg. 30(6), 872-879(2004)

[13]. Ikuko Nishitani, Combustion Products of Moxibustion. The Japan Society of Acupuncture \& Moxibustion. 1987; 37- 3 